\title{
BIBECHANA
}

A Multidisciplinary Journal of Science, Technology and Mathematics ISSN 2091-0762 (Print), 2382-5340 (0nline)

Journal homepage: http://nepjol.info/index.php/BIBECHANA

Publisher: Research Council of Science and Technology, Biratnagar, Nepal

\section{First-principles study of a molecular adsorption of fluorine on monolayer $\mathrm{MoS}_{2}$}

\author{
Hari Paudyal ${ }^{1}$, Nurapati Pantha ${ }^{2}$, Narayan Prasad Adhikari ${ }^{*}$ \\ ${ }^{1}$ Golden Gate international college, Kathmandu, Nepal \\ ${ }^{2}$ Central Department of Physics, Tribhuvan University, Kathmandu, Nepal. \\ "Email: npadhikari@tucdp.edu.np
}

Article history: Received 05 August, 2015; Accepted 12 September, 2015

DOI: http://dx.doi.org/10.3126/bibechana.v13i0.13356

\section{Abstract}

We have performed density functional theory (DFT) based first-principles calculations to study the stability, geometrical structures and electronic properties of pure monolayer Molybdenum disulphide $\left(\mathrm{MoS}_{2}\right)$ and Fluorine $\left(\mathrm{F}_{2}\right)$ adsorbed monolayer $\mathrm{MoS}_{2}$ within the DFT-D2 level of approximations. Present study shows that a $\mathrm{F}_{2}$ molecule adsorbed $\mathrm{MoS}_{2}$ monolayer system is stable. From the geometry and information of adsorption energy of $\mathrm{F}_{2}$ molecule on the different occupation sites of $\mathrm{MoS}_{2}$ monolayer, it has been found that Fluorine prefers atomic adsorption above sulphur (S) atom. The binding energy per equivalent molecular Fluorine above the sulfur (S) atom is found to be $1.83 \mathrm{eV}$. The electronic structure calculations of $\mathrm{MoS}_{2}$ and $\mathrm{F}_{2}$ adsorbed $\mathrm{MoS}_{2}$ monolayer shows that there is a band gap of $1.14 \mathrm{eV}$ and $1.01 \mathrm{eV}$ respectively, at the Fermi level. Further, the symmetry of total DOS for up and down spin calculations reveals that the $\mathrm{F}_{2}$ adsorbed $\mathrm{MoS}_{2}$ monolayer is non-magnetic as similar to that of pure $\mathrm{MoS}_{2}$ monolayer. The projected density of states (PDOS) of a $\mathrm{F}_{2}$ adsorbed $\mathrm{MoS}_{2}$ monolayer is studied to understand the weighted contributions of different orbitals.

CRCOST: All rights reserved.

Keywords: Density functional theory; $\mathrm{MoS}_{2}$ monolayer; band structure properties.

\section{Introduction}

After the discovery of graphene [1], atomic crystals of two-dimensional materials, consisting of single sheets, extracted from bulk materials are gaining considerable attention with a wide range of electronic properties and potential practical applications [2]. The study on low dimensional hexagonal structure based materials have become of great interest to many researchers for nanoscience and consequent nanotechnology. Molybdenum disulfide $\mathrm{MoS}_{2}$, is a two dimensional transition metal dichalcogenide honeycomb structure with certain gap in its electronic bands, consistent with a semiconductor [3]. It is formed by weakly bonded three layers of sulphur, 
molybdenum and sulphur (S-Mo-S). It means that two planes of $\mathrm{S}$ atoms and an intercalated plane of Mo atoms are bound together in a trigonal prismatic arrangement [3,4] of $\mathrm{MoS}_{2}$. Currently, $\mathrm{MoS}_{2}$ is attracting the most valuable researches because of its availability in the form of a natural mineral, molybdenite [5]. Adsorption of foreign atom $/ \mathrm{s}$ and molecule/s on twodimensional materials is a promising approach to suitably modify their properties [6]. The functionalization of $\mathrm{MoS}_{2}$ has also occupied an important domain in the research activities to study electronic, magnetic and band structure properties [7]. A number of theoretical and experimental works have been carried out to study these important behaviors of different elements adsorbed $\mathrm{MoS}_{2}$ monolayer, and have found to yield many interesting results [8].

In the Halogen group (VII), fluorine is the most electronegative and highly reactive element in our periodic table. The primary reason why we choose fluorine in our calculation is that the adsorption of fluorine molecule may have significant potential to modify the electronic properties of $\mathrm{MoS}_{2}$ monolayer, which can be used in many desired electronic practical applications. Further, it may be a useful effort to synthesize chemically modified $\mathrm{MoS}_{2}$ monolayer and present new directions in two dimensional materials. These purpose require the atomistic level of understanding, and in our best knowledge, it is yet to be understood for halogen adsorbed $\mathrm{MoS}_{2}$.

The remaining part of this work is organized in following way. We first discuss the computational details and the systems under our study. We then go into the results section and describe our findings for structural and binding properties of $\mathrm{F} 2$ adsorbed on $\mathrm{MoS}_{2}$ monolayer. The section is followed by the discussion of adsorption properties of F2 on pure $\mathrm{MoS}_{2}$ monolayer. The post-processing analysis, e.g. Density of states is incorporated in the section. The last section, conclusions, summarizes the major findings and possible extension of ongoing research work.

\section{Computational Details}

In the present work, we study the geometrical and electronic properties of monolayer $\mathrm{MoS}_{2}$ and fluorine molecule adsorbed monolayer $\mathrm{MoS}_{2}$. To perform the calculations, we have used Density Functional Theory (DFT) $[9,10]$ implemented in the Quantum ESPRESSO [11] code. The PerdewBurke-Ernzerhof (PBE) variant of the generalized gradient approximation (GGA) [12], incorporating van der Walls interaction via Grimme's model [13, 14] is used to treat electronelectron interaction. The algorithm has used Rappe-Rabe-Kaxiras-Joannopoulos (RRKJ) model of ultra- soft pseudo-potentials to account theinteraction between the ion cores and valence electrons. The plane wave expansion with kinetic energy cut-off values of magnitudes $35 \mathrm{Ry}$, and $350 \mathrm{Ry}$, respectively, are used for wave-function and charge density. A mesh of $10 \times 10 \times 1 \mathrm{k}$-points is used for unit cell of $\mathrm{MoS}_{2}$ monolayer for Brillouin-zone integration and a mesh of $4 \times 4 \times 1 \mathrm{k}$-points is taken for $3 \times 3$ supercell of monolayer $\mathrm{MoS}_{2}$. Further, in order to minimize the interactions between two adjacent MoS2 layers, a vaccum distance of $12 \AA$ is maintained in the calculations. All the structures are optimized using the BFGS (Broyden-Fletcher-Goldfarb-Shanno) [15] scheme until the total energy 
change is less than $10^{-4}$ Ry between two consecutive scf steps and force acting is less than $10^{-3}$ Ry/Bohrs. The 'Marzarri-vanderbilt' [16] smearing or cold smearing with a small broadening width of $0.001 \mathrm{Ry}$ is used. Furthermore, diagonalization method is chosen as 'david', with mixing factor 0.6 for self-consistency. Spin polarized calculations are accommodated to study the magnetic properties of the systems. For the density of states (DOS) calculations of pure monolayer MoS2 and F2 adsorbed monolayer MoS2 systems, we have used $3 \times 3$ supercell of monolayer MoS2 with a denser mesh of $(8 \times 8 \times 1)$ k-points.

\section{Results and Discussion}

A hexagonal unit cell of monolayer $\mathrm{MoS}_{2}$ with the basis of three (1-Mo and 2-S) atoms in honeycomb lattice structure is initially constructed by using experimental value [17]. It is build up by single layers of S-Mo-S atoms as shown in figure 1. It consists of two planes of Sulphur (S) atoms and an intercalated plane of Molybdenum (Mo) atoms bound with the sulphur atoms in a trigonal prismatic arrangement. Each Mo atom is surrounded by six first-nearest neighbouring $\mathrm{S}$ atoms, while each $S$ atom is connected to three first-nearest neighbouring Mo atoms [3, 5] as shown in figure 1.

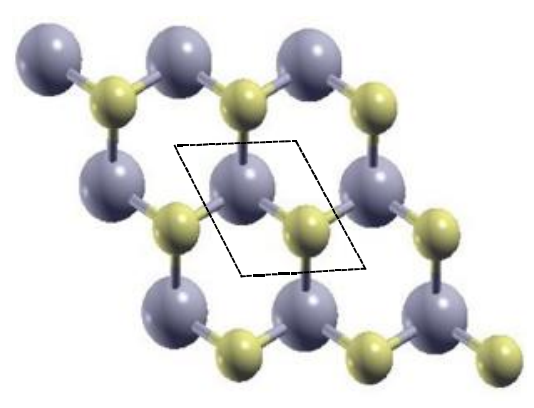

(a)

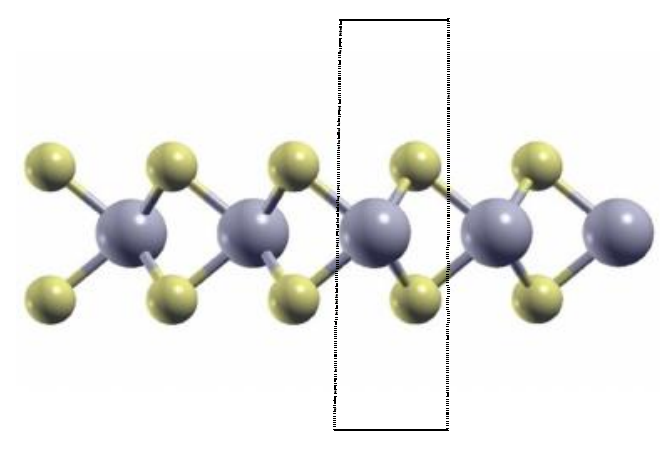

(b)

Fig. 1: Unit cell of monolayer $\mathrm{MoS}_{2}$ (shown by dotted parallelogram), (a) Top-view and (b) Sideview. The unit-cell consists of a single Mo atom intercalated between two $S$ atoms at a bond length of $2.42 \AA$ and S-MO-S angle of $80.80^{\circ}$. Propagation of $\mathrm{MoS}_{2}$ unit-cell along x- and y-directions to form $3 \times 3$ supercell of monolayer $\mathrm{MoS}_{2}$. Single Mo-atom is covalently bonded with $6 \mathrm{~S}$-atoms to form hexagonal honey-comb structure.

After the construction of the unit-cell, the structure is optimized with respect to latticeparameter ' $a$ ', kinetic energy cutoff ( $\mathrm{E}_{c u t-o f f)}$ for plane wave and the number of k-points along $\mathrm{x}$ - and $\mathrm{y}$-axes respectively. Based on these convergence tests, we obtained that the lattice constant ' $a$ ' for the unit cell of monolayer $\mathrm{MoS}_{2}$ is $3.18 \AA$, which agrees with the previous results [3,5]. For the $3 \times 3$ supercell of monolayer $\mathrm{MoS}_{2}$ the lattice constant is three times that of the unit cell. Further, the plot of the total energy verses the number of k-points shows that the energy of the unitcell of monolayer $\mathrm{MoS}_{2}$ is almost constant when the number of $\mathrm{k}$ points $\left(n_{k x}=n_{k y}\right)$ crosses 10. A a mesh of $10 \times 10 \times 1 \mathrm{k}$-points is, therefore, used for Brillouin-zone integration of unit cells and a mesh of $4 \times 4 \times 1$ k-points are taken for $3 \times 3$ supercell. A plane wave basis set with energy cutoff values of 
$35 \mathrm{Ry}$ for the wave-function and $350 \mathrm{Ry}$ for the charge density is used for the expansion of the ground state electronic wave functions.

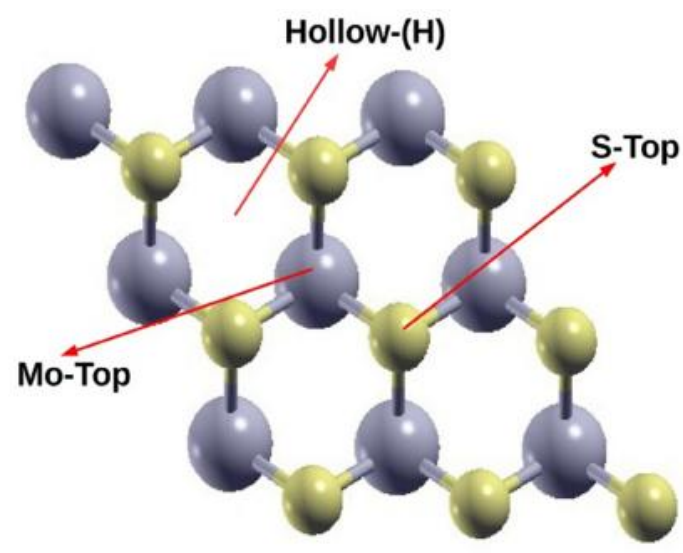

(a)

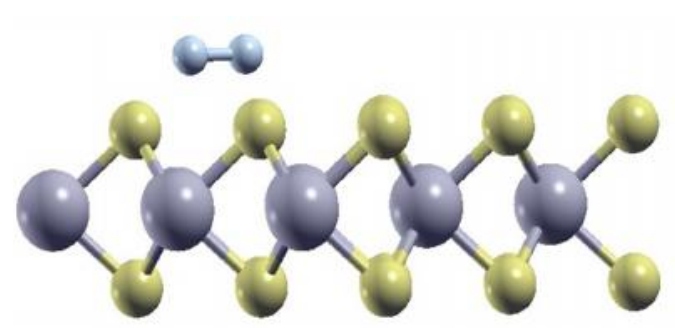

(b)

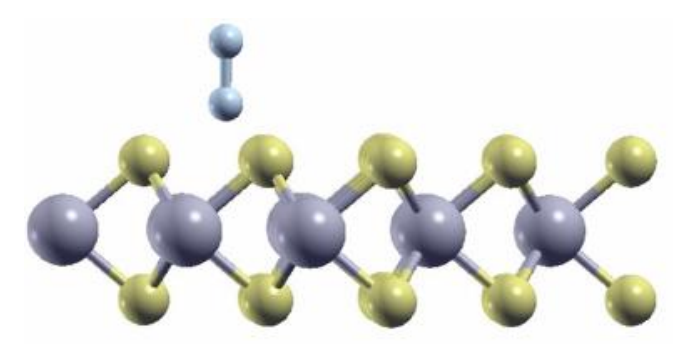

(c)

Fig. 2: Initial structures of $\mathrm{MoS}_{2}$ and $\mathrm{F}_{2}$ added $\mathrm{MoS}_{2}$. (a) Different possible adsorption sites, (b) horizontal (parallel) configuration of molecular $\mathrm{F}_{2}$ and (c) vertical (perpendicular) configuration of molecular $\mathrm{F}_{2}$ for the adsorption of molecular $\mathrm{F}_{2}$ on monolayer $\mathrm{MoS}_{2}$.

A molecular fluorine $\left(\mathrm{F}_{2}\right)$ is adsorbed on monolayer $\mathrm{MoS}_{2}$ of size $3 \times 3$ supercell. The adsorption is carried out at different possible sites of $\mathrm{MoS}_{2}$ (figure 2(a)), with different orientations/configurations of F2 molecule. We have taken three different occupation sites for the adsorption, which are: the Hollow (H) site at the center of the hexagonal ring, the top of the Mo atom (Mo-Top) site, and the top of the S atom (S-Top). Further, two configurations of molecular $\mathrm{F}_{2}$ for its adsorption, i.e., the vertical (perpendicular) to the $\mathrm{MoS}_{2}$ plane and the horizontal (parallel) to the $\mathrm{MoS}_{2}$ plane, are considered. All together we have studied six different possible ways for its adsorption. For each of the sites and the configurations, the total energy and hence the binding energy (B.E) of the adsorbed molecule is calculated. The most suitable site with the suitable configuration is determined by using the information of total energy (minimum) or the binding energy (maximum). 

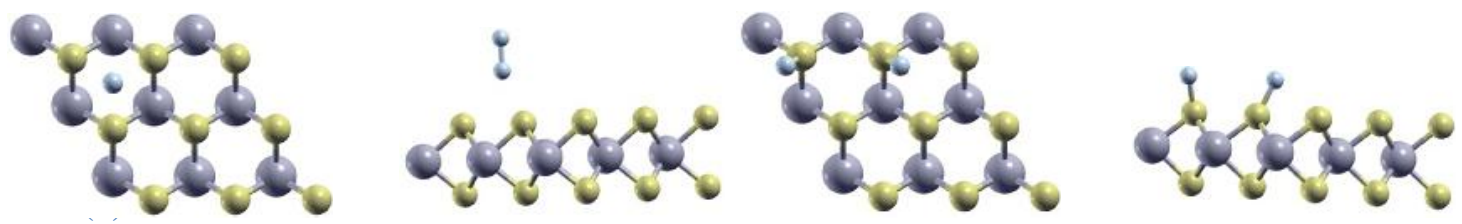

(a)

(b)
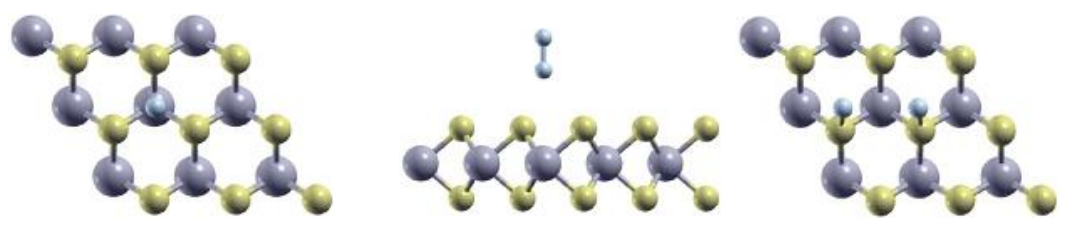

(c)

\author{
(d)
}
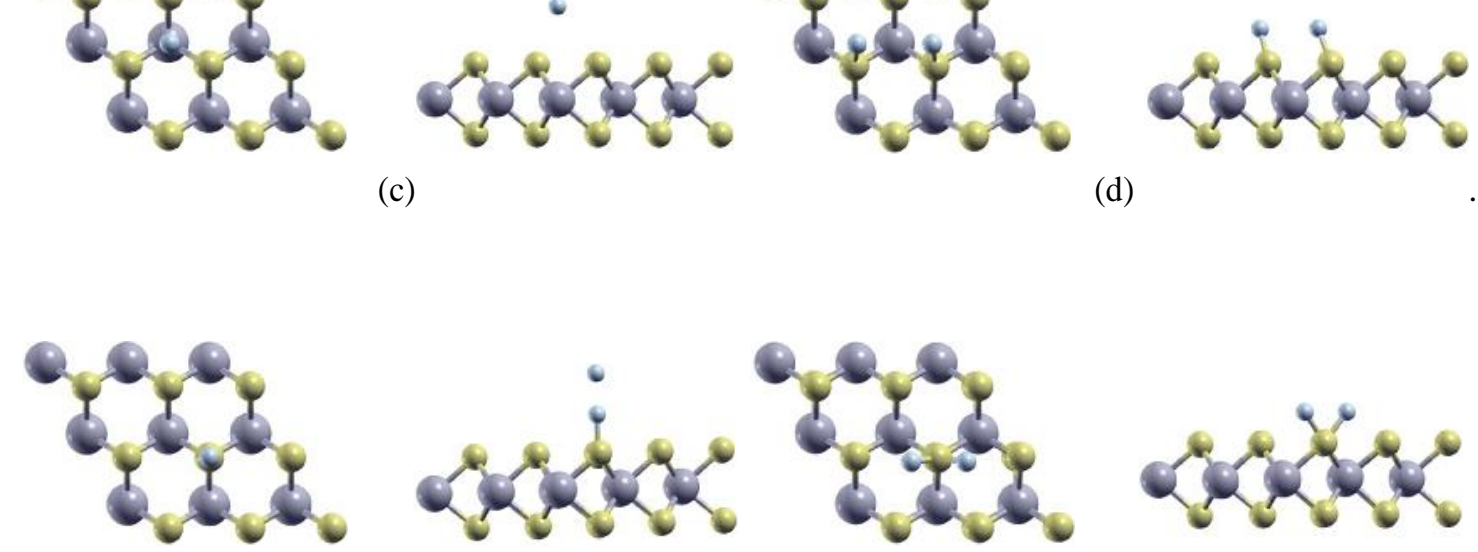

(e)

(f)

Fig. 3: Optimized geometries of $F_{2}$ molecule adsorbed monolayer $\mathrm{MoS}_{2}$ with top- and side-view respectively, (a) $F_{2}$ molecule perpendicular to $\mathrm{MoS}_{2}$ plane at $\mathrm{H}$-site $\left(\mathrm{H}\right.$ - perpendicular), (b) $F_{2}$ molecule parallel to $\mathrm{MoS}_{2}$ plane at $\mathrm{H}$ site (H-parallel), (c) $F_{2}$ molecule perpendicular to $\mathrm{MoS}_{2}$ plane at Mo-T site (Mo-T-perpendicular), (d) $F_{2}$ molecule parallel to $\mathrm{MoS}_{2}$ plane at Mo$\mathrm{T}$ site (Mo-T-parallel), (e) $F_{2}$ molecule perpendicular to $\mathrm{MoS}_{2}$ plane at S-T site (S-Tperpendicular) and (f) $F_{2}$ molecule parallel to $\mathrm{MoS}_{2}$ plane at S-T site (S-T-parallel)

The Binding Energy (B.E)/adsorption energy $(\Delta E)$ of a molecular fluorine on monolayer $\mathrm{MoS}_{2}$ is calculated using the relation,

$$
\Delta E=E_{F_{2}}+E_{M_{o S}}-E_{M o S_{2}+F_{2}}
$$

where, $E_{F_{2}}, E_{\mathrm{MoS}_{2}}$ and ${ }_{E_{M o S_{2}+F_{2}}}$ are the total energies of the isolated $\mathrm{F} 2$ molecule, mono- layer $\mathrm{MoS}_{2}$, and the $\mathrm{F}_{2}$ adsorbed monolayer $\mathrm{MoS}_{2}$ system respectively. Of the three ad- sorption sites $(\mathrm{H}, \mathrm{Mo}-\mathrm{T}$ and S-T), with two possible configurations taken, the site with the largest adsorption energy is referred as the suitable site for adsorption. The optimized structures of $F_{2}$ adsorbed monolayer $\mathrm{MoS}_{2}$ systems are shown in the figure 3. 
Table 1: The table lists the adsorption energy of molecular Fluorine $(\Delta \mathrm{E})$, it's height from the average of upper sulphur plane (h), optimized F-F distance in $F_{2}$ molecule $\left(d_{F-F}\right)$ after adsorption, the distance of the atomic $\mathrm{F}$ from it's nearest S-atom $\left(d_{S-F}\right)$ and the distortion observed in $3 \times 3$ supercell of monolayer $\mathrm{MoS}_{2}\left(d_{\mathrm{MoS}_{2}}\right)$ due to adsorption of $F_{2}$.

\begin{tabular}{|c|l|c|c|c|c|c|}
\hline Adsorption site & Configuration of $\mathrm{F} 2$ & $\Delta \mathrm{E}(\mathrm{eV})$ & $\mathrm{h}(\AA)$ & $d_{F-F}(\AA)$ & $d_{S-F}(\AA)$ & $d_{M o S_{2}}\left(10^{-1} \AA\right)$ \\
\hline Hollow (H) & Perpendicular & 0.1788 & 3.187 & 1.486 & 3.031 & -0.823 \\
& Parallel & 1.4661 & 1.634 & 4.487 & 1.791 & -0.139 \\
& & & & & & \\
\hline Mo-Top & Perpendicular & 0.1455 & 3.358 & 1.464 & 3.191 & -0.943 \\
& Parallel & 0.9916 & 1.582 & 2.979 & 1.764 & -0.135 \\
& & & & & & \\
\hline S-Top & Perpendicular & 0.6327 & 2.657 & 1.827 & 1.827 & -0.039 \\
& Parallel & 1.8304 & 1.396 & 2.159 & 1.729 & 0.366 \\
& & & & & & \\
\hline
\end{tabular}

Table 1 represents the adsorption/binding energy values $(\Delta \mathrm{E})$, the relaxed distance ofcenter of mass of molecular F2 from S-plane of monolayer $\mathrm{MoS}_{2}(\mathrm{~h})$, the separation be- tween the two $\mathrm{F}$ atoms in $\mathrm{F}_{2}\left(d_{F-F}\right)$, the distance of a $\mathrm{F}$ atom from nearest $\mathrm{S}$-atom $\left(d_{S-F}\right)$ and the distortion observed in $3 \times 3$ supercell of monolayer $\mathrm{MoS}_{2}\left(d_{\mathrm{MoS}_{2}}\right)$ due to adsorption of $\mathrm{F}_{2}$. The table shows that a $\mathrm{F}_{2}$ molecule is bound to all the adsorption sites considered and the parallel configuration on the top of the $\mathrm{S}$ atom is the most stable one among them. There is a large variation in the binding energy values of molecular fluorine, which is due the different configurations of $F_{2}$ molecule towards the varying sites of substrate. During the optimization of the adsorbed system it is found that the molecular fluorine, in some of the cases, breaks it's bond and each of the isolated F atom gets bonded with the nearest $\mathrm{S}$ atom. The geometries with the atomic adsorption of $\mathrm{F}$ are seen more stable (see table 1). The table also reveals that the system having maximum B.E has the minimum planer distance from the S-plane. In addition, the distance between two $\mathrm{F}$ atoms increases when it is bound more strongly to monolayer $\mathrm{MoS}_{2}$. The results imply that the boding of $\mathrm{F} / \mathrm{F}_{2}$ with the substrate happens on the cost of weakening of F-F interaction.

The Density of States (DOS) of up and down spin for pure and $\mathrm{F}_{2}$ adsorbed $\mathrm{MoS}_{2}$, with reference to Fermi level (represented by vertical dotted line), are shown in the figures 4(a) and 4(b). Present calculations show that the Fermi energy for the pure and $\mathrm{F}_{2}$ adsorbedmonolayer $\mathrm{MoS}_{2}$ are 0.5647 $\mathrm{eV}$ and $-0.0461 \mathrm{eV}$, respectively. The values (of Fermi energy) imply that there is a shift in Fermi level by $0.6108 \mathrm{eV}$ after the adsorption of $\mathrm{F}_{2}$. The density of states for pure monolayer $\mathrm{MoS}_{2}$ can also reveal that there is a gap (between valence and conduction bands) of about $1.14 \mathrm{eV}$ near in the Fermi level. Since the PBE type GGA calculations underestimate the band gap near by 
the Fermi level $[18,19]$, the value of the band gap obtained from the present calculations is smaller than the previously reported values [3,4]. However, the electronic band structure (figure is not shown) reveals that the material is direct band gap semiconductor, which is consistent with the core concept of the band structure reported in the references. Further, the symmetry in the up- and down-DOS supports the fact that the monolayer $\mathrm{MoS}_{2}$ is non-magnetic material.

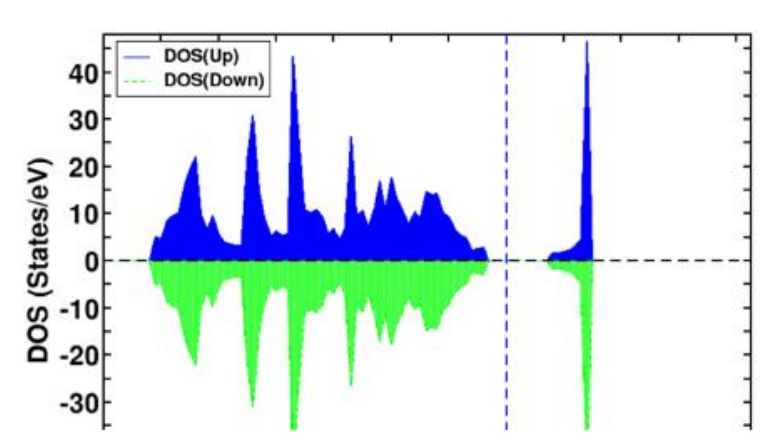

(a)

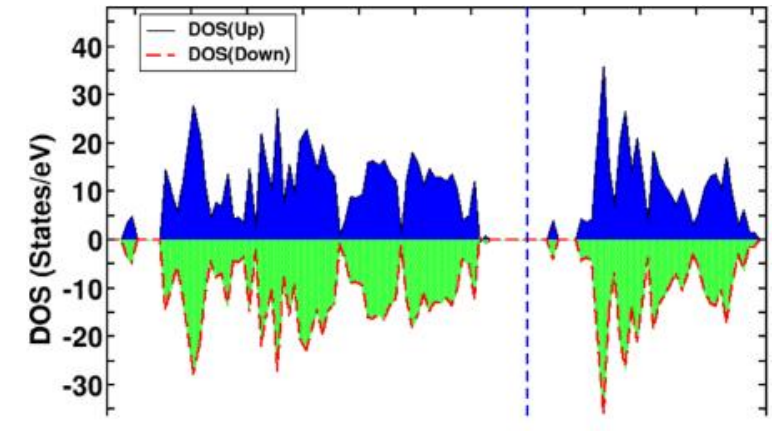

(b)

Fig. 4: Density of states (DOS) of (a) monolayer $\mathrm{MoS}_{2}$, and (b) $\mathrm{F}_{2}$ adsorbed monolayer $\mathrm{MoS}_{2}$.

On the other hand, the band gap near the Fermi level of $\mathrm{F}_{2}$ adsorbed monolayer $\mathrm{MoS}_{2}$ is found to be $1.01 \mathrm{eV}$. It means that the band gap has decreased by $0.13 \mathrm{eV}$ due to adsorption of $\mathrm{F}_{2}$. It may be due to the fact that fluorine is highly reactive and most electronegative element in the nature, and certain bands at valence/conduction band are occupied by the addition of $\mathrm{F}_{2}$ electrons. The high peaks which are seen in total DOS of pure monolayer $\mathrm{MoS}_{2}$ are found to be decreasing after the adsorption of $F_{2}$. The symmetry of DOS (for up- and down-DOS) of $F_{2}$ adsorbed monolayer $\mathrm{MoS}_{2}$ shows that the system remains non-magnetic after the adsorption of $\mathrm{F}_{2}$ as well.

To get the better understanding of the sharp energy bands on total DOS of the $\mathrm{F}_{2}$ molecule adsorbed monolayer $\mathrm{MoS}_{2}$, we have performed the partial DOS analysis (PDOS). The contributions of different orbitals of Mo, $\mathrm{S}$ and $\mathrm{F}$ atoms on the $\mathrm{F}_{2}$ molecule adsorbed $\mathrm{MoS}_{2}$ are presented in the figures 5, 6 and 7. In the figures the Fermi energy is taken as reference, and represented by the vertical dotted line. The figures reveal that $4 \mathrm{~d}, 3 \mathrm{p}$ and $2 \mathrm{p}$ orbitals of Mo, S and F atoms respectively, are the dominant contributions on the sharp energy bands of total DOS. 

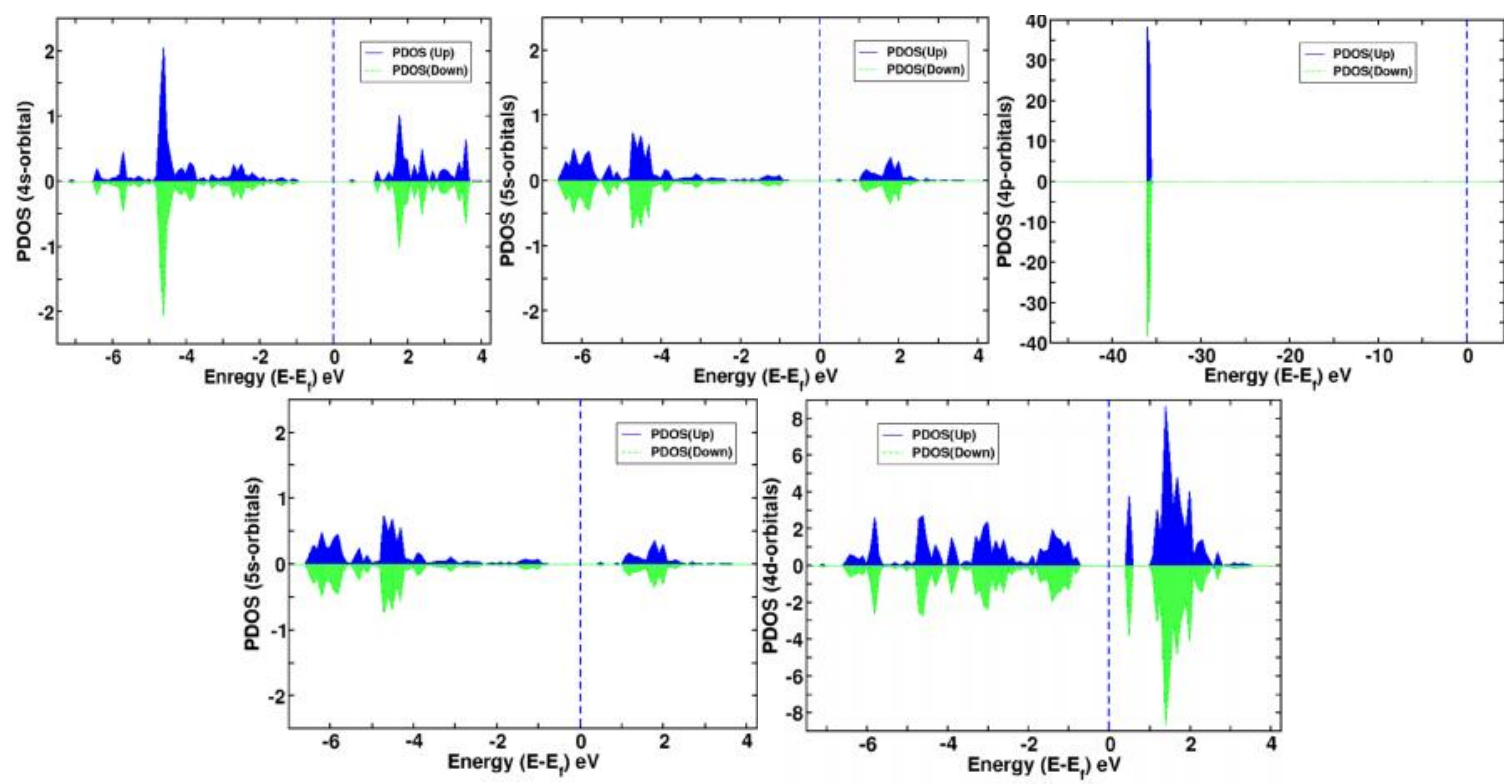

Fig. 5: PDOS of $4 s, 5 s, 4 p, 5 p$ and $4 d$ orbitals of Mo atom respectively. PDOS due to $4 d$-orbitals is seen dominant over the others.
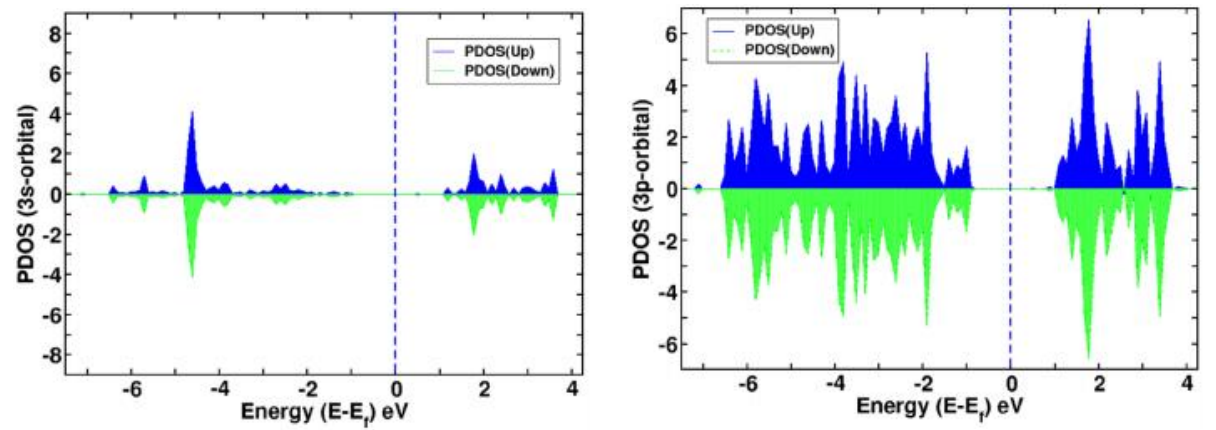

Fig. 6: PDOS of $3 s$ and $3 p$ orbitals of $S$ atom, respectively. PDOS due to 3p-orbitals, poisoned nearby Fermi level, is seen dominant.
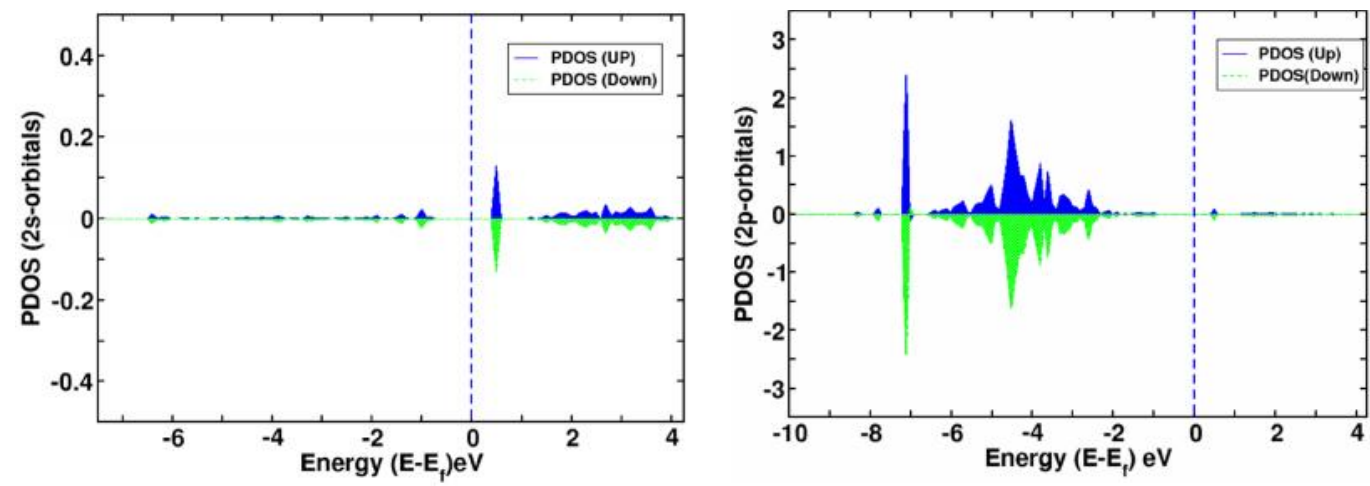

Fig. 7: PDOS of $2 s$ and $2 p$ orbitals of $F$ atoms respectively. The PDOS due to $2 p$-orbitals is seen dominant over the 2 s-orbitals. 


\section{Conclusions}

The structural and electronic properties of pure and F2 adsorbed monolayer MoS2 systems are studied. The binding energy values of F2 at different sites suggest that F2 can be adsorbed on monolayer MoS2 and the parallel configuration of F2 above sulphur (S) atom is found to be the most stable with the binding energy of $1.83 \mathrm{eV}$. The geometry reveals that the molecular F2 dissociates and each atom of $\mathrm{F}$ is adsorbed to nearby $\mathrm{S}$ in the structure. The DOS calculations of the pure monolayer MoS2 find a band gap of $1.14 \mathrm{eV}$ and reiterates that the system is nonmagnetic. In case of F2 adsorbed monolayer MoS2, the band gap decreases to $1.03 \mathrm{eV}$. Up- and down-DOS are again symmetrical, and indicate that the F2 adsorbed monolayer MoS2 is also non-magnetic.

\section{Acknowledgements}

We acknowledge the partial support from The Abdus Salam International Center for Theoretical Physics (ICTP) through office of external activities within NET-56 project. Also, we extend our gratitude to The World Academy of Science and Technology (TWAS) research grants for providing computational facilities.

\section{References}

[1] K. S. Novoselov, A. K. Geim, S. V. Morozov, D. Jiang, M. I. Katsnelson, S. V. Dubonos, I. V. Grigorieva, A. A. Firsov, Nature, 438 (2005) 197. http://dx.doi.org/10.1038/nature04233

[2] E. L. Wolf, Application of Graphene, Springer, Brooklyn, USA, 2014. http://dx.doi.org/10.1007/978-3-319-03946-6

[3] E.S.Kadantsev, P.Hawryla, Sol. St. Comm., 152 (2012) 909-913. http://dx.doi.org/10.1016/j.ssc.2012.02.005

[4] S. Lebegue and O. Eriksson, Phy. Rev. B, 79 (2009) 115409. http://dx.doi.org/10.1103/PhysRevB.79.115409

[5] D. Lembke, S. Bertolazzi, A. Kis, Acc. Chem. Res., 1(2015) 48. http://dx.doi.org/10.1021/ar500274q

[6] T. P. Kaloni, R. P. Joshi, N. P. Adhikari and U. Schwingenschlogl, Appl. Phys. Lett., 104 (2014) 073116. http://dx.doi.org/10.1063/1.4866383

[7] Q. Yue, S. Chang, S. Qin and J. Li Phy. Lett. A., 377 (2013) 1362-1367. http://dx.doi.org/10.1038/ncomms7298

[8] Y. Wang, B. Wang, R. Huang, B. Gao, F. Kong and Q. Zhang Physica E., 63 (2014) 276-282. http://dx.doi.org/10.1063/1.4922419

[9] P. Hohenberg and W. Kohn, Phys. Rev. B, 136 (1964) 864. http://dx.doi.org/10.1103/PhysRev.136.B864

[10] W. Kohn and L. J. Sahm, Phys. Rev. , 140(1965) 1133. http://dx.doi.org/10.1103/PhysRev.140.A1133 
[11] P. Giannozzi, S. Baroni, N. Bonini, M. Calandra, R. Car, C. Cavazzoni, et al., J. Phys., Condens. Matter, $21(2009) 395502$.

http://dx.doi.org/10.1088/0953-8984/21/39/

[12] J. P. Perdew, K. Burke, M. Ernzerhof, Phys. Rev. Lett., 77 (1996) 566.

http://dx.doi.org/10.1103/PhysRevLett.77.3865

[13] S. Grimme, J. Comput. Chem., 25 (2004) 1463.

http://dx.doi.org/10.1002/jcc. 20078

[14] S. Grimme, J. Comput. Chem. 27 (2006)1787.

http://dx.doi.org/10.1002/jcc.20495

[15]B. G. Pfrommer, M. Cote, S. G. Louie, M. L. Cohen, J. Comput. Phys., 131 (1997) 233. http://dx.doi.org/10.1103/PhysRevB.80.235418

[16] N. Marzari, D. Vanderbilt, A. De Vita, and M. C. Payne, Phys. Rev. lett., 82 (1999) 3296. http://dx.doi.org/10.1103/PhysRevLett.82.3296

[17] K. Novoselov, D. Jiang, F. Schedin, T.Booth, V.Khotkevich, S.Morozov and A. Geim, Proc. Natl. Acad. Sci. U.S.A., 102 (2005) 1045.

http://dx.doi.org/10.1073/pnas.0502848102

[18] F. Karlicky, R.Zboril and M. Otypka , J. Chem. Phys., 137 (2012) 034709. http://dx.doi.org/10.1063/1.4736998

[19] X. Li, S. Wu, S. Zhou and Z. Zhu, Nano. Res. Let., 9 (2014) 110. http://dx.doi.org/10.1186/1556-276X-9-110 